\title{
Design of High-Voltage Switch-Mode Power Amplifier Based on Digital-Controlled Hybrid Multilevel Converter
}

\author{
Yanbin Hou, Wanrong Sun, Aifeng Ren, and Shuming Liu \\ School of Electronic Engineering, Xidian University, Xian 710071, China \\ Correspondence should be addressed to Yanbin Hou; ybhou@mail.xidian.edu.cn
}

Received 20 April 2016; Revised 18 August 2016; Accepted 25 August 2016

Academic Editor: Wei-Zen Chen

Copyright ( 2016 Yanbin Hou et al. This is an open access article distributed under the Creative Commons Attribution License, which permits unrestricted use, distribution, and reproduction in any medium, provided the original work is properly cited.

\begin{abstract}
Compared with conventional Class-A, Class-B, and Class-AB amplifiers, Class-D amplifier, also known as switching amplifier, employs pulse width modulation (PWM) technology and solid-state switching devices, capable of achieving much higher efficiency. However, PWM-based switching amplifier is usually designed for low-voltage application, offering a maximum output voltage of several hundred Volts. Therefore, a step-up transformer is indispensably adopted in PWM-based Class-D amplifier to produce highvoltage output. In this paper, a switching amplifier without step-up transformer is developed based on digital pulse step modulation (PSM) and hybrid multilevel converter. Under the control of input signal, cascaded power converters with separate DC sources operate in PSM switch mode to directly generate high-voltage and high-power output. The relevant topological structure, operating principle, and design scheme are introduced. Finally, a prototype system is built, which can provide power up to 1400 Watts and peak voltage up to \pm 1700 Volts. And the performance, including efficiency, linearity, and distortion, is evaluated by experimental tests.
\end{abstract}

\section{Introduction}

Class-A, Class-B, and Class-AB amplifiers are usually termed as linear amplifiers for their switching devices operate in linear mode, while Class-D amplifiers are also known as switching amplifiers, in which the switching devices are either fully turned on or completely turned off, operating in switch mode. This means that when the switch is conducting (turned on) there is virtually no voltage across the switch and that when the switch is not conducting (turned off) there is no current flowing through the switch. So the ideal efficiency of Class-D amplifier is $100 \%$ in theory [1]. In fact, the commonly used semiconductor switching devices, such as IGBT (Insulated Gate Bipolar Transistor) and MOSFET (Metal Oxide Semiconductor Field Effect Transistor), have saturation voltage drop or on-state resistance when they are turned on. Although part of power has been consumed as heat by switches, efficiencies over $85 \%$ can be achieved in most situations for Class-D amplifier. By comparison, linear amplifier has a theoretical efficiency of 78\% at the utmost [2].

In a typical Class-D amplifier, input signal is converted into a series of pulses through pulse width modulation
(PWM), which serve as control signals for driving power switches. The switching devices together with DC sources realize power and voltage amplification. At the output stage of Class-D amplifier, low-frequency amplified signal is retrieved across the load by filtering out high-frequency carrier wave. Due to its single half-bridge or full-bridge topology, PWMbased switching amplifier usually outputs a peak voltage of dozens or hundreds of Volts [3]. Step-up transformer seems to be a ready-made solution to boost up output voltage, since existing PWM-based switching amplifiers can provide enough output power. However, the noisy and heavy midfrequency step-up transformer will make the whole amplifier system bulky and expensive. What is worse, the insertion of step-up transformer between amplifier and load will introduce additional power loss, thus reducing the overall system efficiency. One of our outside partners is confronted with such a dilemma. They built an analog PWM-based switching amplifier and used a bulky step-up transformer to produce the rated voltage of 1000 Volts and rated power of 1000 Watts across a $1000-\mathrm{Ohm}$ resistor over the operating frequency range of 100-1000 Hertz. But the average system efficiency is about $70 \%$, presenting a challenge to cool. What 


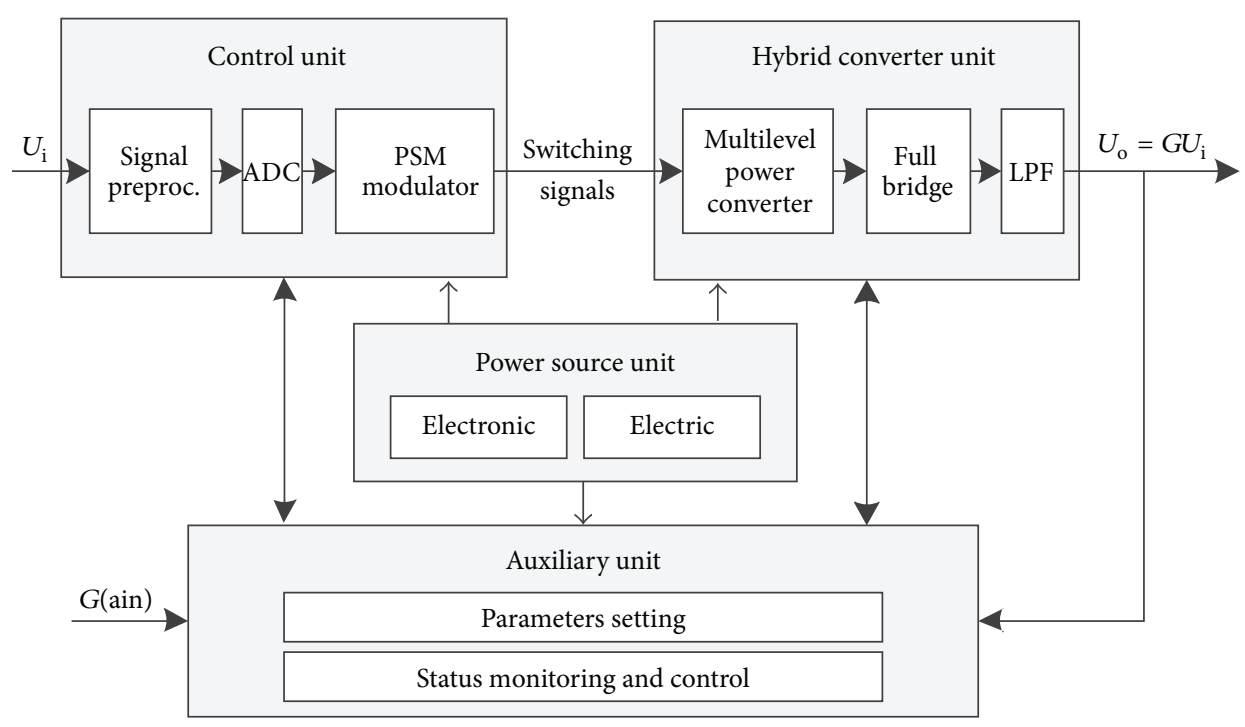

FIGURE 1: Block diagram of high-voltage switching amplifier.

is more, the bulky step-up transformer has a weight of over 50 kilograms, almost two-thirds of the gross weight. Our target is to rebuild a switching amplifier with main requirements as follows:

Output voltage up to 1000 Vrms.

Output power up to $1000 \mathrm{~W}$.

Overall efficiency above $85 \%$.

Output distortion below $2.5 \%$.

Gross weight less than $80 \mathrm{Kg}$.

The requirements about high efficiency and light weight exclude the possibility of using step-up transformer to boost up voltage. From the view of power electronics, the desired switching amplifier can be considered as a programmable high-voltage power supply operating in switch mode under the control of input signal. NPC- (neutral point clamped-) based and cascaded H-bridge (full-bridge) multilevel converters are two commonly used topologies to directly produce high-voltage output $[4,5]$. The former uses a single highvoltage DC source and multiple cascaded semiconductor switches together with clamping diodes or flying capacitors, usually involving complicated voltage balancing measures [6-8]. The latter adopts modular design, of which each converter consists of a low-voltage DC source and four semiconductor switches. Thus it will demand a great number of semiconductor switches and control signals when employing multiple cascaded full-bridge converters to generate highvoltage output.

In order to directly generate high-voltage output and reduce power semiconductor switches as well, a new circuit architecture, called hybrid multilevel converter, is proposed in this paper. The corresponding control scheme is designed on the basis of pulse step modulation (PSM) by adopting digital signal processing technology. Section 2 introduces the design scheme, Section 3 gives the performance tests, and
Section 4 is the conclusion. Experimental results show that this new system can provide up to 1400 Watts and \pm 1700 Volts peak voltage with high efficiency and low distortion as expected.

\section{Design of High-Voltage Switching Amplifier}

Figure 1 shows the functional block diagram of high-voltage switch-mode power amplifier developed in this paper.

As shown in Figure 1, this switching amplifier consists of four units, which are control unit, hybrid converter unit, power source unit, and auxiliary unit, respectively. The workflow is as follows.

Small analog input signal is firstly preprocessed and then digitalized by a 12-bit analog-to-digital converter (ADC). According to the ADC data and user settings, PSM modulator generates a set of switching signals, which are further isolated and driven before applying to hybrid converter unit. Under the control of corresponding switching signals, multilevel power converter transforms energy from separate low-voltage DC sources to augment modulated switching signals and full bridge takes charge of the switchover between positive and negative phase. At the final output stage, a lowpass filter is used to retrieve the expected amplified output waveform.

For the sake of efficiency and reliability, the whole system was developed utilizing digital signal processing technology. PSM modulator was implemented with a high-performance floating-point digital signal processor (DSP) and a highvolume field programmable gate array (FPGA). Auxiliary unit adopted digital technologies at the most extent, like human-machine interface (HMI) for setting gain and other parameters, measuring output voltage and current, monitoring temperatures, and so on.

The following sections introduce key points in detail to develop a $\pm 1700 \mathrm{Vp} / 1400 \mathrm{~W}$ switch-mode power amplifier, of 


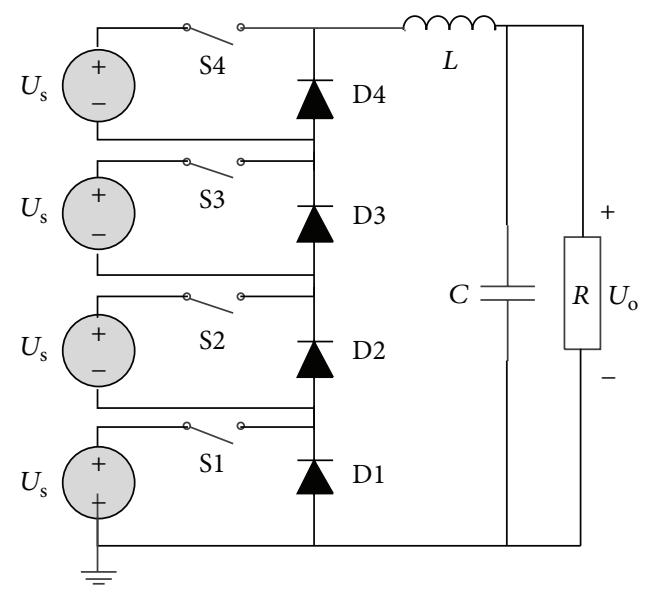

(a)

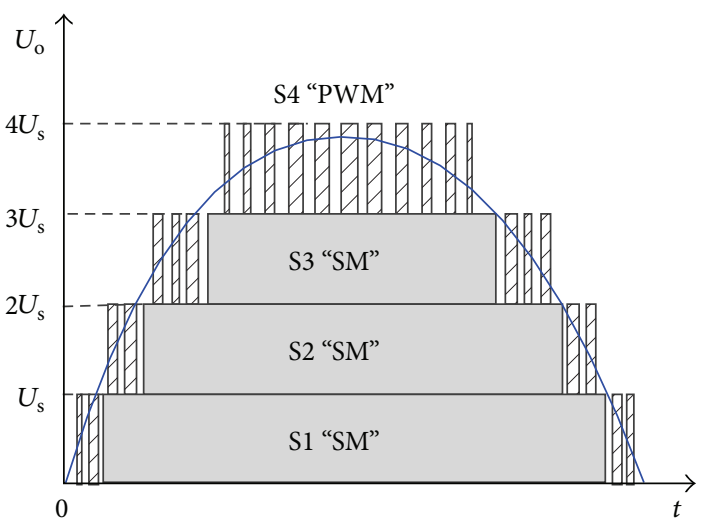

(b)

Figure 2: PSM-based HVPS. (a) Schematic and (b) principle.

which efficiency is expected to be no less than $85 \%$ with low distortion in the midfrequency range $(100-1000 \mathrm{~Hz})$.

\subsection{Pulse Step Modulation. Brown Boveri Corporation pre-} sented pulse step modulation (PSM) and applied it broadly in short-wave transmitter and high-voltage power supply (HVPS) [9-11]. Figure 2 illustrates the schematic circuit and work principle of PSM-based HVPS to quadruple its output voltage of each converter.

As shown in Figure 2(a), a PSM-based HVPS basically consists of multiple (four in this example) power converters connected in series, which work in switch mode under the control of input reference signal (here taking semi-sine wave as an example). Each power converter has a separate low-voltage DC source $U_{\mathrm{s}}$, a semiconductor switch $\mathrm{S}$, and a free-wheeling diode $\mathrm{D}$ [12]. The diode is to provide a low impedance path when the corresponding converter is switched off, allowing current from other working converters to pass through. Regardless of voltage drops across diodes and switches, total output voltage $U_{0}$ is simply equal to several times of unit step voltage $U_{s}$, depending on the number of switched-on power inverters. Obviously, actual output voltage is just a roughly stepwise approximation to the expected waveform, which is referred to as step modulation (SM). To further refine output, pulse width modulation (PWM) and LC components are adopted to smooth the transition from one step to the other. Taking Figure 2(a) as an example, let switches S1 S3 operate in SM mode and let switch S4 operate in PWM mode; the filtered output voltage $U_{\mathrm{o}}$ across the load is plotted as a blue solid line in Figure 2(b).

As stated above, PSM consists of coarse SM and fine PWM. Figure 2(b) shows that each SM operates at a rather low frequency the same as input reference signal. It is also noticed that PWM need to switch much faster than SM to achieve an accurate representation of the input signal. Following Nyquist theorem, PWM need to run at least twice the maximum input frequency, but actual design generally adopts a much higher ratio (typically 10 to 50) for reducing distortion [13]. In theory, high-frequency PWM can improve output waveform. However, switch loss increases as switching becomes faster. Generally, PWM will take a compromise between output waveform and switch loss, depending on the practical application.

2.2. Hybrid Converter Unit. As illustrated in Figure 2, PSMbased HVPS can only produce unipolar voltage. In order to operate as an amplifier, circuit needs to be further modified. Conventional method is that every converter adopts fullbridge architecture, employing four switches and two groups switching signals to produce $0 \sim \pm U_{s}$. However, it will increase system cost and control complexity if many full-bridge converters are used to produce high voltage.

In this paper, a hybrid multilevel converter was designed, in which information on amplitude and phase of input signal is separately modulated. As shown in Figure 3, eight cascaded power converters (PC1 PC8) produce desired amplitude while full bridge (composed of Q1 Q4) controls phase. Compared with cascaded full-bridge converters, this hybrid architecture employs much fewer switches and control signals.

Power converters PC1 PC8 are identical, except for corresponding to different switching signals. Each one consists of separated $220 \mathrm{~V} / 3 \mathrm{~A}$ DC source $U_{\mathrm{s}}$, semiconductor switch $\mathrm{S}$, and free-wheeling diode $\mathrm{D}$. In consideration of switching speed and power loss, semiconductor switches S1 S8 all use power MOSFETs, which have small on-state resistance. Freewheeling diodes D1 D8 adopt fast recovery diodes. Intensive simulations and tests confirm that MOSFET and diode suffer the maximum potential difference of $U_{\mathrm{s}}$ in all situations, so many power MOSFETs available can be used, such as IRF840, 2SK1507, and FMV11N60E. 2SK1507 with a typical drainsource on-state resistance of $R_{\mathrm{DS}(\mathrm{on})}=0.85 \Omega$ was adopted. Diodes adopted MUR860 with a maximum instantaneous forward voltage drop $V_{\mathrm{F}}=1.5 \mathrm{~V}$. Switches S1 S7 and S8 are 


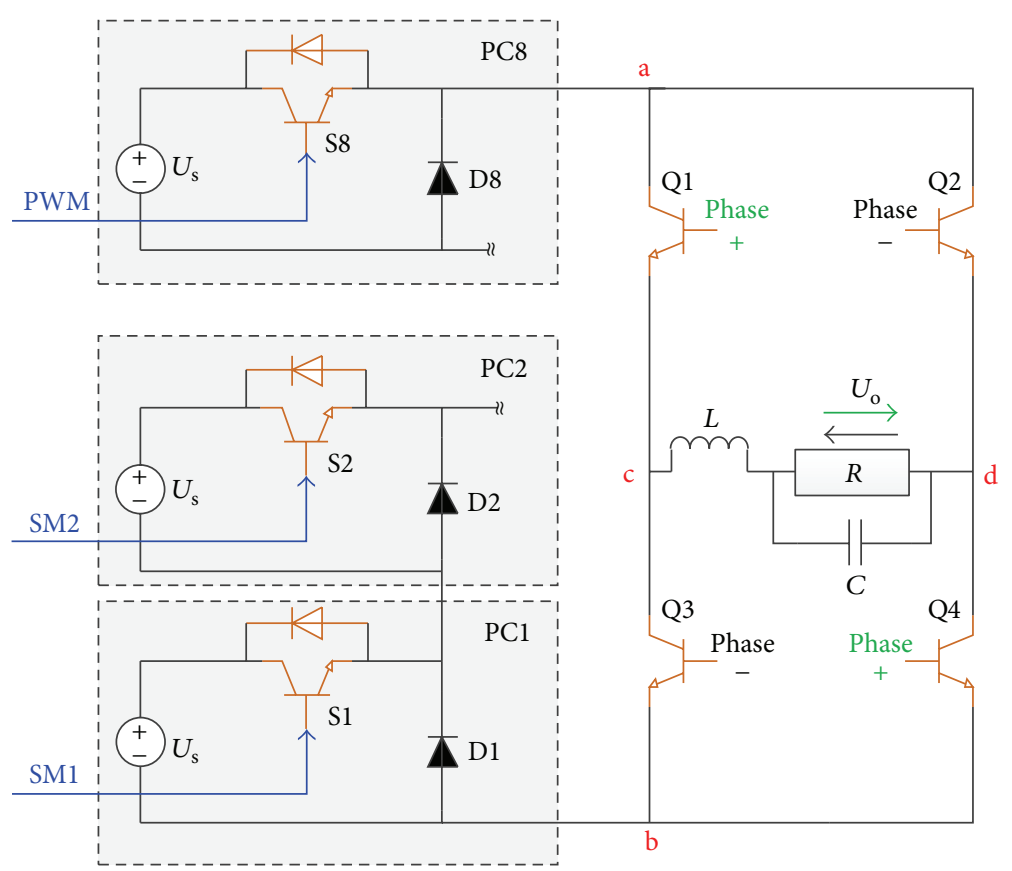

FIGURE 3: Schematic of hybrid multilevel converter.

under the control of switching signals SM1 SM7 and PWM, respectively.

The full bridge is built with four discrete IGBTs Q1 Q4, under the control of switching signals Phase+/Phase-. When the input signal is nonnegative, Q2 and Q3 are switched off simultaneously; meanwhile Q1 and Q4 are switched on simultaneously and vice versa. Although ZVS (Zero Voltage Switching) is applied, dead time is also inserted between commutations to avoid potential shoot-through risk. IGBTs adopted IXYS IXBH12N300 with a maximum saturation voltage drop $V_{\mathrm{CE} \text { (sat) }}=3.2 \mathrm{~V}$.

Inductor $L$, capacitor $C$, and resistive load $R$ make up a Butterworth low-pass filter (LPF) to recover the amplified output signal from high-voltage modulated waveform. Given load resistance and cut-off frequency, inductance and capacitance can be obtained by

$$
\begin{gathered}
L=\frac{R}{\left(\sqrt{2} \pi f_{C}\right)}, \\
C=\frac{1}{\left(2 \sqrt{2} \pi R f_{C}\right)},
\end{gathered}
$$

where $f_{C}$ denotes the cut-off frequency, $f_{C}$ in Hertz, $L$ in Henrys, $C$ in Faradays, and $R$ in Ohms, respectively.

The LPF filter has a $-3 \mathrm{~dB}$ cut-off frequency of $5 \mathrm{kHz}$, corresponding to $R=1 \mathrm{k} \Omega, L=44 \mathrm{mH}$, and $C=18 \mathrm{nF}$.

2.3. Digital PSM Modulator. Unlike carrier-based PWM that adopts analog modulation [14], we designed digital PSM modulator to generate SM and PWM switching signals by the following formula:

$$
\begin{aligned}
M(n) & =\operatorname{fix}\left(\frac{G\left|U_{\mathrm{i}}(n)\right|}{U_{\mathrm{s}}}\right) \\
d(n) & =\operatorname{rem}\left(\frac{G\left|U_{\mathrm{i}}(n)\right|}{U_{\mathrm{s}}}\right) \\
\text { Phase+ } & = \begin{cases}\mathrm{ON}, & \text { if } U_{\mathrm{i}}(n) \geq 0 \\
\text { OFF, } & \text { if } U_{\mathrm{i}}(n)<0\end{cases} \\
\text { Phase- } & = \begin{cases}\text { ON, } & \text { if } U_{\mathrm{i}}(n)<0 \\
\text { OFF, } & \text { if } U_{\mathrm{i}}(n) \geq 0\end{cases}
\end{aligned}
$$

$$
n=t_{1}, t_{2}, \ldots, t_{n},
$$

where $\left|U_{i}(n)\right|$ is the absolute amplitude of sampled input signal at moment $t_{n}$. $G$ is predefined voltage gain; $U_{s}$ is DC source voltage. Function fix $(\cdot)$ rounds the data towards zero, resulting in an integer $M(n)$, which is the number of power converters switched on between $t_{n}$ and $t_{n+1}$. Function $\operatorname{rem}(\cdot)$ retrieves the remainder after division, so $d(n)$ is a decimal, representing the "on" time of a power converter relative to sampling interval; that is, $d(n)$ determines the duty cycle of PWM switching signal (e.g., 0.50 is equal to $50 \%$ ). Phase+ and Phase- record the phase information about sampled input signal, serving as the switching signals for full bridge.

In other words, during sampling interval between $t_{n}$ and $t_{n+1}, M(n)$ power converters work in SM mode, and one power converter operates in PWM mode with a duty cycle of $d(n)$. Obviously, the switching frequency of PWM is equal 
to the sampling frequency of ADC. Here we adopted unipolar centre-aligned PWM, whose advantages and implementation could be referred to [15].

2.4. Software Simulation. The workflow of the above switching amplifier is demonstrated through simulation. For example, a sine wave of $1 \mathrm{kHz}$ and $\pm 1 \mathrm{Vp}$ needs to be amplified by a voltage gain of $G=800$. It is assumed that sampling frequency of $\mathrm{ADC}$ is $40 \mathrm{kSa} / \mathrm{s}$, so that there will be 40 sampled points in a cycle, as shown in Figure 4(a). According to (2), if $U_{s}=220 \mathrm{Vdc}$, it needs that three converters work in SM mode and one converter operates in PWM mode. Digital PSM modulator generates switching signals, as plotted in Figure 4(b). The unused converters are always switched off, which are not plotted in Figure 4(b). Figure 4(c) gives the modulated amplitude after amplification, measured between points a and b (marked in Figure 3). Figure 4(d) shows the output voltages after adding phase information and filtering, measured between points $\mathrm{c}$ and $\mathrm{d}$ (marked in Figure 3), in which the blue solid line represents modulated waveform after amplification, and the red dotted line is the final output voltage across load after LPF demodulation.

In this simulation, zero-crossing points of input signal are all sampled by ADC. However, this may be not true in most actual situations. So ZVS will be applied to force all SM and PWM signals to be off at the moments of commutation, for example, when $t=0.5,1.0,1.5$, and $2.0 \mathrm{~ms}$ in Figure 4(b).

It is important to note that IGBTs usually take more time to be switched on or off compared to MOSFETs due to finite switching speed. In order to protect full bridge at the moment of voltage phase alternation, proper dead time is added to ensure that short circuit will not happen between points a and b (marked in Figure 3), which means that both phase control signals in Figure 4(b) are switched off during the dead time.

\section{Experimental Tests and Results}

3.1. Prototype System. Based on the above introduction, a high-voltage switch-mode power amplifier is developed, as shown in Figure 5.

From top to down, there are main distribution box, electronic case for control unit and auxiliary unit, electric case for hybrid converter unit, and two 4-channel AC-DC $220 \mathrm{~V}$ power sources in a 19 -inch standard cabinet $(600 \mathrm{~mm}$ $\times 600 \mathrm{~mm} \times 1050 \mathrm{~mm}, \mathrm{~W} \times \mathrm{D} \times \mathrm{H})$. A resistor of $1000 \mathrm{Ohms}$ with cooling equipment is used as dummy load, laid outside the cabinet for facilitating heat dissipation.

3.2. Total Harmonic Distortion. Many factors in switching amplifier can cause distortion, such as power switches, nonlinearity of output LPF, DC source voltage fluctuations, dead time, and modulation technologies. As one of the most common and important features, total harmonic distortion (THD) is usually used to evaluate system distortion. Given that input signal is a sine wave, THD is most commonly defined as the ratio of the rms amplitude of a set of harmonic

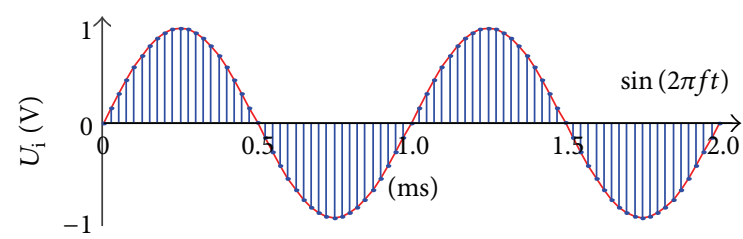

(a)
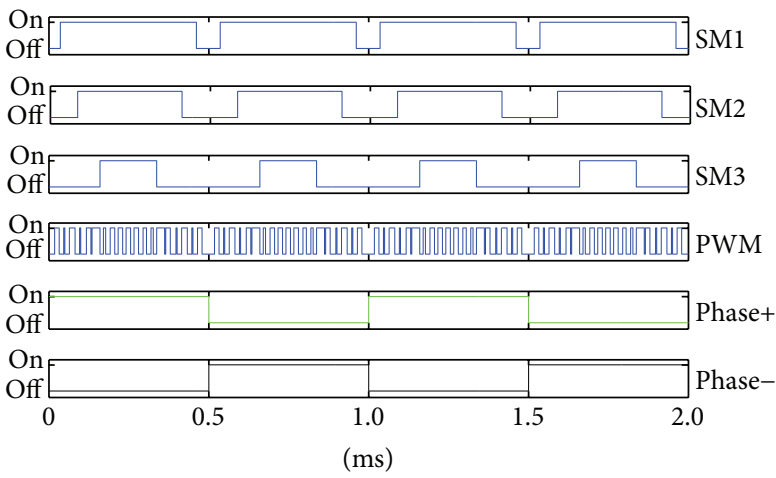

(b)

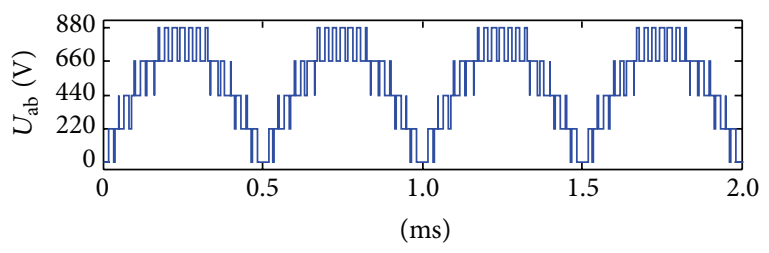

(c)

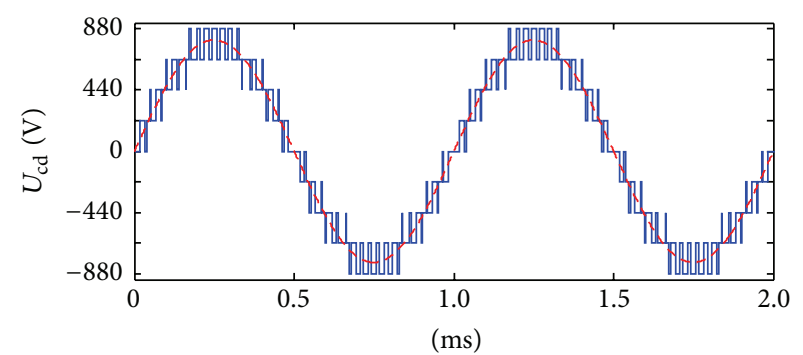

- Unfiltered
-- Filtered

(d)

FIGURE 4: Simulation of switching power amplifier. (a) Sampled input signal, (b) switching signals for power converters and full bridge, and ((c) and (d)) voltage waveforms measured between a$\mathrm{b}$ and $\mathrm{c}-\mathrm{d}$ points, respectively (points a-d marked in Figure 3 ).

frequencies to that of fundamental frequency, which can be formulated as follows [16]:

$$
\mathrm{THD}=\frac{\sqrt{U_{2}^{2}+U_{3}^{2}+\cdots+U_{n}^{2}}}{U_{1}},
$$

where $U_{1}$ denotes the rms voltage of fundamental frequency and $U_{n}$ is the rms voltage of the $n$th harmonic frequency.

Sine wave of 1 Vrms with frequency sweeping from 100 to $1000 \mathrm{~Hz}$ was used as input signal to investigate the prototype system. Figure 6 shows the screenshots of output waveforms 
TABLE 1: THD values of power amplifier system.

\begin{tabular}{lcccc}
\hline \multirow{2}{*}{ Freq. $(\mathrm{Hz})$} & \multicolumn{4}{c}{ Output power $(\mathrm{W})$} \\
& 300 & 650 & 960 & 1300 \\
\hline 100 & $1.52 \%$ & $1.47 \%$ & $1.06 \%$ & $1.04 \%$ \\
200 & $1.59 \%$ & $1.53 \%$ & $1.12 \%$ & $1.05 \%$ \\
300 & $1.65 \%$ & $1.52 \%$ & $1.15 \%$ & $1.04 \%$ \\
400 & $1.73 \%$ & $1.58 \%$ & $1.23 \%$ & $1.05 \%$ \\
500 & $1.68 \%$ & $1.58 \%$ & $1.26 \%$ & $1.09 \%$ \\
600 & $1.69 \%$ & $1.60 \%$ & $1.27 \%$ & $1.19 \%$ \\
800 & $1.71 \%$ & $1.66 \%$ & $1.33 \%$ & $1.30 \%$ \\
1000 & $1.77 \%$ & $1.76 \%$ & $1.40 \%$ & $1.34 \%$ \\
\hline
\end{tabular}

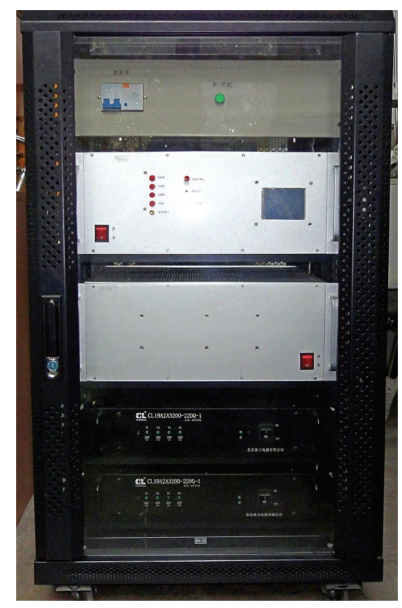

Figure 5: Photo of power amplifier system.

at frequencies of 100, 500, and $1000 \mathrm{~Hz}$. On the oscilloscope display, the upper channel shows output voltage and the lower one is its corresponding fast Fourier transform (FFT). It is noticed that the FFT spectrum is plotted as magnitude in $\mathrm{dB}$ relative to $1 \mathrm{Vrms}$; the corresponding rms voltage is retrieved by

$$
U_{n}=10^{d B_{n} / 20},
$$

where $d B_{n}$ denotes the voltage gain relative to $1 \mathrm{Vrms}$ at the $n$th harmonic frequency and $U_{n}$ is the rms output voltage of the $n$th harmonic frequency.

Table 1 lists THD values measured at four different output power values. Without regard to individual measurement errors, it can be found that THD value increases as signal frequency rises and that THD value decreases as output power increases. It is not difficult to explain this phenomenon. With input signal sweeping from low frequency to high frequency, the sampled points in a single cycle reduce, resulting in output waveform not so smooth as before. When output power increases, signal-to-noise ratio is improved to a certain degree.

It is noticed that measured output power in Table 1 is an average of output power values measured from 100 to $1000 \mathrm{~Hz}$ with a step size of $100 \mathrm{~Hz}$, keeping input signal amplitude
TABLE 2: Efficiency of power amplifier system.

\begin{tabular}{lcc}
\hline$P_{\mathrm{c}}(\mathrm{W})$ & $P_{\mathrm{o}}(\mathrm{W})$ & Efficiency \\
\hline 350 & 300 & $85.7 \%$ \\
712 & 650 & $91.3 \%$ \\
1022 & 960 & $93.9 \%$ \\
1361 & 1300 & $95.5 \%$ \\
\hline
\end{tabular}

constant. Averaging is aimed at diminishing measurement error for high voltage. A typical example is shown in Figure 6. With the same input signal and amplification gain, peak and rms output voltages fluctuated irregularly at different frequencies.

3.3. The Maximum Output. To measure the maximum output power, sine wave of $1 \mathrm{kHz}$ is used as input signal and its amplitude increases until output distortion reaches THD = $10 \%$. Figure 7 shows output voltage when amplifier outputs the maximum power of about $1500 \mathrm{~W}$.

As shown in Figure 7, waveform appears apparent clipping at the maximum output power value. For the sake of practicability, the maximum output voltage is limited to $3400 \mathrm{Vpp}$ (a critical point of appearing voltage clipping), and the corresponding maximum output power is hence lowered to about $1400 \mathrm{~W}$, estimated by

$$
P_{\mathrm{o}}=\frac{1}{8 R} U_{\mathrm{pp}}{ }^{2}=\frac{1}{8 \times 1000} \times(3400 \mathrm{~V})^{2}=1445 \text { Watts. }
$$

3.4. Efficiency and Linearity. The efficiency corresponding to the output power in Table 1 is listed in Table 2. $P_{\mathrm{c}}$ represents the whole power consumed by entire amplifier system; $P_{\mathrm{o}}$ is the output power measured on load. It is noticed that $P_{\mathrm{o}}$ is an average of output power values measured from 100 to $1000 \mathrm{~Hz}$ with a step size of $100 \mathrm{~Hz}$, as the same reason explained in Section 3.2.

As for an amplifier, nonlinearity caused by gain variation will affect the waveform shape of analog output with respect to the corresponding analog input. Figure 8 plots three groups of output versus input signal amplitude with a constant voltage gain of $G=1000$.

As shown in Figure 8, the output approximately follows a linear relationship relative to the input. The average voltage gains measured at frequencies of 400,800 , and $1000 \mathrm{~Hz}$ are 1027,1036 , and 1021, respectively. That is to say, this new developed high-voltage switch-mode power amplifier has a statistical linearity error less than $4 \%$.

3.5. Automatic Protection. The running status of power amplifier system is under real-time surveillance, including output voltage, output current, output power, and system temperature. Once any alarm about overvoltage, overcurrent, or overtemperature is triggered, amplifier system will be shut down within $1 \mathrm{~ms}$, as illustrated in Figure 9. In the oscilloscope screenshot, Channel 1 records alarm signal, and Channel 2 is recording switching signal. If a failure occurs, falling edge of alarm signal is first detected and low level 


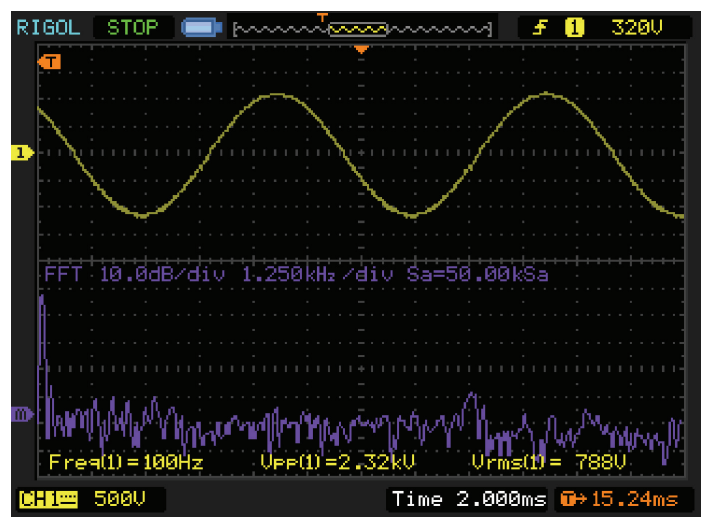

(a)

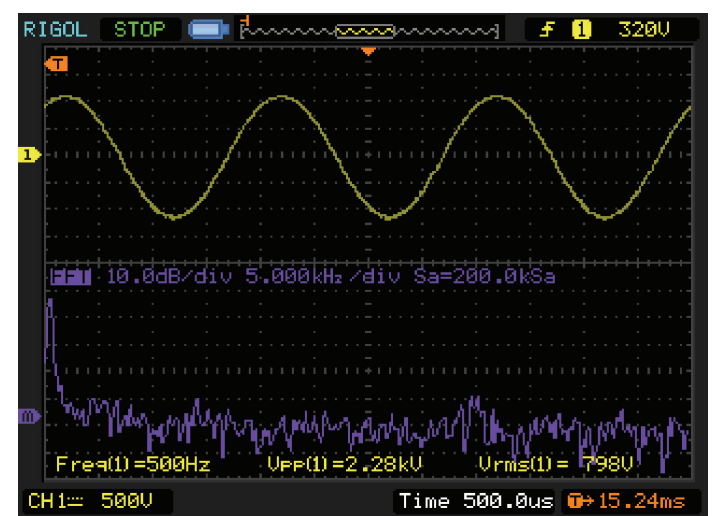

(b)

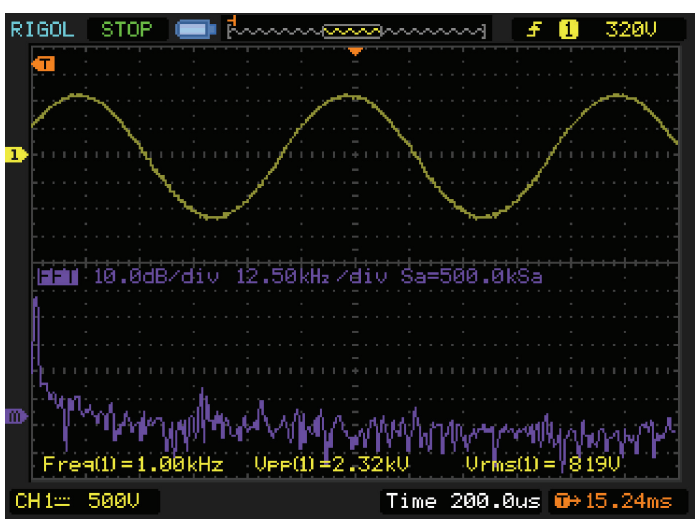

(c)

Figure 6: Oscilloscope screenshots of output voltage at frequencies of (a) $100 \mathrm{~Hz}$ ( $500 \mathrm{~V} / \mathrm{div}, 2 \mathrm{~ms} / \mathrm{div})$, (b) $500 \mathrm{~Hz}$ (500 V/div, $500 \mathrm{us} / \mathrm{div}$ ), and (c) $1000 \mathrm{~Hz}$ (500 V/div, $200 \mathrm{us} /$ div).

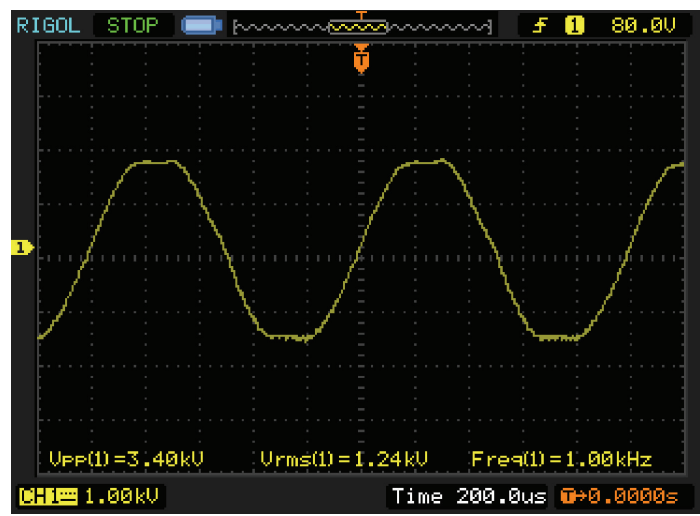

FIGURE 7: High-voltage output waveform of clipping with THD = $10 \%$ ( $1 \mathrm{kV} /$ div, $200 \mathrm{us} /$ div).

of alarm signal is then reconfirmed within about 600 us. If failure does happen, control unit will switch off all power converters one by one within 40 us.

3.6. Overall Comparison. Table 3 gives an overall comparison between the new digital PSM-based switching amplifier and the existing analog PWM-based switching amplifier. The

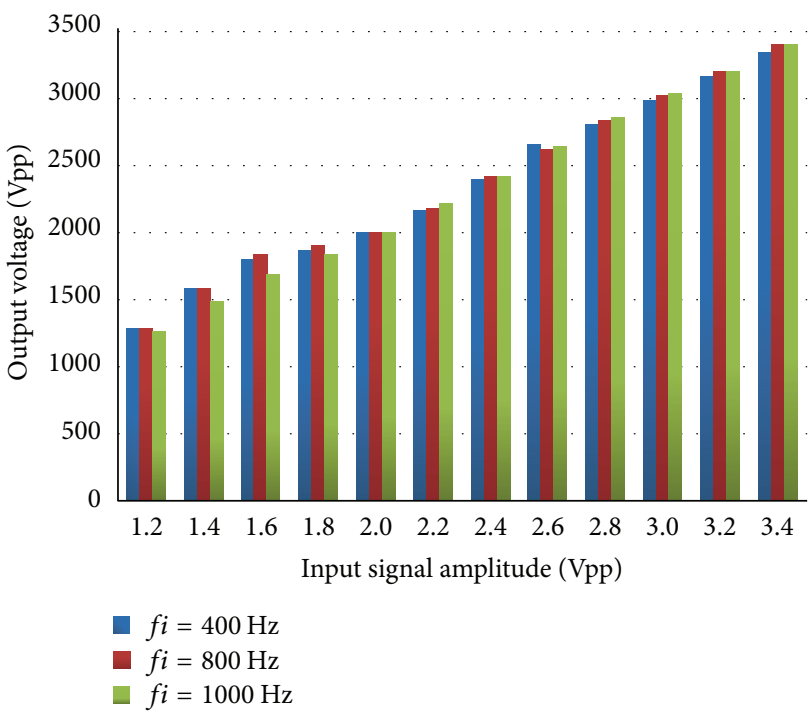

FIGURE 8: Linearity of power amplifier system.

specification and performance of the latter are provided by our partner off campus, including modulation technology, circuit topology, output voltage and power, distortion, 


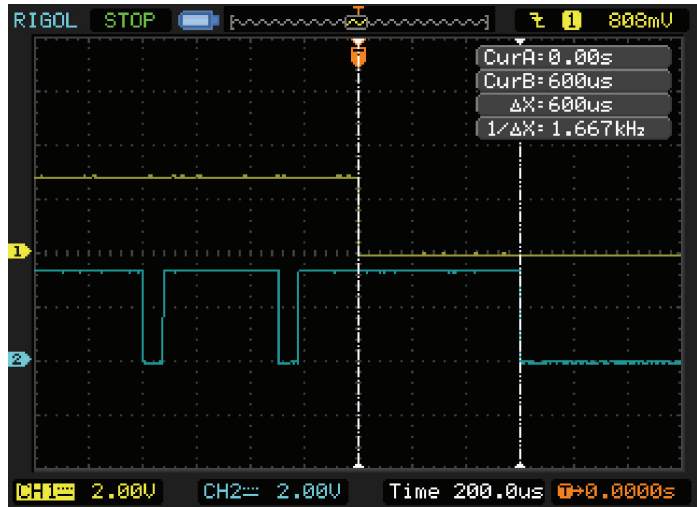

(a)

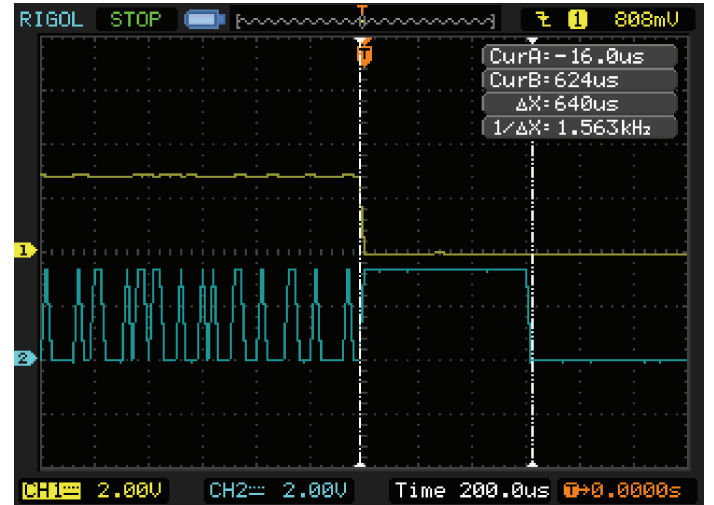

(b)

FIGURE 9: Oscilloscope screenshots of automatic protection. (a) CH1: failure alarm signal and CH2: first closed SM1 signal. (b) CH1: failure alarm and CH2: last closed PWM signal (2 V/div, 200 us/div).

efficiency, and weight. For convenience, the two switching amplifiers are labelled as PSM-based amplifier and PWMbased amplifier, respectively.

In PWM-based switching amplifier, a high-speed analog comparator compares a triangular carrier wave of $300 \mathrm{KHz}$ with the input signal to generate PWM switching signals. But, in PSM-based switching amplifier as described in Section 2.4, the corresponding PWM switching signal is $40 \mathrm{KHz}$. High PWM switching frequency means that more sampled points are used to approximate the input signal, thus yielding smooth waveform and low distortion. Furthermore, PWMbased switching amplifier only adopts fast power MOSFETs in the half-bridge stage, while PSM-based switching amplifier adopts IGBTs with much higher blocking voltage in the full-bridge stage. But IGBT switches slower than MOSFET due to tail-current effect at turn-off. Therefore, PWM-based switching amplifier can generate better output waveform. However, the dependence of PWM-based amplifier on stepup transformer lowers overall efficiency and increases gross weight.

\section{Conclusion}

In this paper, a switch-mode power amplifier based on digital pulse step modulation and hybrid multilevel converter is designed to directly produce high-voltage output without the aid of step-up transformer.

Compared with available PWM-based switching amplifier coupled with a step-up transformer, this new developed system can provide output power up to 1400 Watts and peak voltage up to \pm 1700 Volts with encouraging efficiency and portability. The major advantages are its hybrid architecture and digital modulation. When directly generating highvoltage output, a lot of control signals and power switches are cut down by modulating the information on amplitude and phase of input signal separately.

However, there is further work to improve this design.
TABle 3: Comparisons of two power amplifier systems.

\begin{tabular}{lcc}
\hline Items & PSM-based amplifier & PWM-based amplifier \\
\hline Modulation & Digital PSM & Analog PWM \\
Circuit topology & Multilevel & Half bridge \\
Power supplies & DC 220 V & DC $\pm 80 \mathrm{~V}$ \\
Step-up transformer & No need & Need \\
Maximum output & $\pm 1700 \mathrm{Vp}$ & $\pm 1500 \mathrm{Vp}$ \\
& $1400 \mathrm{~W}$ & $1200 \mathrm{~W}$ \\
THD $^{*}$ & $1.38 \%$ & $0.85 \%$ \\
Efficiency* & $94.2 \%$ & $82.5 \%$ \\
Gross weight & $50 \mathrm{Kg}$ & $80 \mathrm{Kg}$ \\
User interface & Touch LCD & LED indicators \\
\hline
\end{tabular}

${ }^{*}$ The test condition is outputting $1000 \mathrm{Vrms}$ sine at $1 \mathrm{KHz}$.

Firstly, switching frequency needs to be increased for more practical applications. However, MOSFETs in highvoltage converter cannot switch at a frequency as high (300 kHz or above) as they do in PWM-based audio amplifier, because this will lead to serious overshoot and EMI problems. Thus, more elaborate modulation scheme needs to be considered, such as phase-shifting modulation technologies.

Secondly, more intelligent strategies should be adopted. For example, redundant power converters can timely replace the failed ones without shutdown. If there is no usable power converter, amplifier will appropriately lower output voltage to prevent serious distortion.

\section{Competing Interests}

The authors declare that there are no competing interests regarding the publication of this paper.

\section{Acknowledgments}

This research is supported by the Fundamental Research Funds for the Central Universities under Grant no. JB140205. 


\section{References}

[1] M. Bloechl, M. Bataineh, and D. Harrell, "Class D switching power amplifiers: theory, design, and performance," in Proceedings of the IEEE SoutheastCon 2004, pp. 123-146, Greensboro, NC, USA, March 2004.

[2] M. Berkhout and L. Dooper, "Class-D audio amplifiers in mobile applications," IEEE Transactions on Circuits and Systems, vol. 57, no. 5, pp. 992-1002, 2010.

[3] D. Self, Audio Power Amplifier Design Handbook, Focal Press, Boston, Mass, USA, 5th edition, 2009.

[4] F. J. Liu, Modern Inverter Technology and Application, Publishing House of Electronics Industry, Beijing, China, 2006 (Chinese).

[5] V. Sala, R. Salehi, M. Moreno-Eguilaz, M. Salehifar, and L. Romeral, "Clamping diode caused distortion in multilevel NPC Full-Bridge audio power amplifiers," in Proceedings of the 38th Annual Conference on IEEE Industrial Electronics Society (IECON '12), pp. 4941-4948, IEEE, Montreal, Canada, October 2012.

[6] W. W. He, P. Palmer, X. Q. Zhang, M. Snook, and Z. H. Wang, "IGBT series connection under active voltage control," in Proceedings of the 14th European Conference on Power Electronics and Applications (EPE '11), pp. 1-9, IEEE, Birmingham, UK, September 2011.

[7] P. Palmer, W. W. He, X. Q. Zhang, J. Zhang, and M. Snook, "IGBT series connection under Active Voltage Control with temporary clamp," in Proceedings of the 38th Annual Conference on IEEE Industrial Electronics Society (IECON '12), pp. 465-470, Montreal, Canada, October 2012.

[8] T. Lu, Z. M. Zhao, S. Q. Ji et al., "Design of voltage balancing control circuit for series connected HV-IGBTs," in Proceedings of the 16th International Conference on Electrical Machines and Systems (ICEMS '13), pp. 515-518, IEEE, Busan, South Korea, October 2013.

[9] W. Schminke, "The merits of modern technology for today's high power short-wave transmitters," IEEE Transactions on Broadcasting, vol. 34, no. 2, pp. 126-133, 1988.

[10] J. Alex and W. Schminke, "A high voltage power supply for negative ion NBI based on PSM technology," in Proceedings of the 17th IEEE/NPSS Symposium on Fusion Engineering (SOFE '97), vol. 2, pp. 1063-1066, IEEE, San Diego, Calif, USA, October 1997.

[11] L. Y. Yao, Y. Q. Wang, X. H. Mao, Y. L. Wang, and Q. Li, “A fully digital controller of high-voltage power supply for ECRH system on HL-2A," IEEE Transactions on Plasma Science, vol. 40, no. 3, pp. 793-797, 2012.

[12] J. Alex and W. Schminke, "Fast switching, modular high-voltage DC/AC-power supplies for RF-amplifiers and other applications," in Proceedings of the 16th IEEE/NPSS Symposium on Fusion Engineering (SOFE '95), pp. 936-939, IEEE, Champaign, Ill, USA, October 1995.

[13] M. J. Hawksford, "Modulation and system techniques in PWM and SDM switching amplifiers," Journal of the Audio Engineering Society, vol. 54, no. 3, pp. 107-139, 2006.

[14] M. H. Rashid, Power Electronics Handbook-Devices, Circuits, and Applications, Butterworth-Heinemann, Boston, Mass, USA, 3rd edition, 2011.

[15] B. Lei, G.-C. Xiao, and X.-L. Wu, "Comparison of performance between bipolar and unipolar double-frequency sinusoidal pulse width modulation in a digitally controlled $\mathrm{H}$-bridge inverter system," Chinese Physics B, vol. 22, no. 6, Article ID 060509, pp. 281-288, 2013.

[16] D. Shmilovitz, "On the definition of total harmonic distortion and its effect on measurement interpretation," IEEE Transactions on Power Delivery, vol. 20, no. 1, pp. 526-528, 2005. 


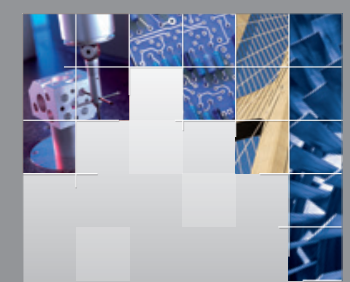

\section{Enfincering}
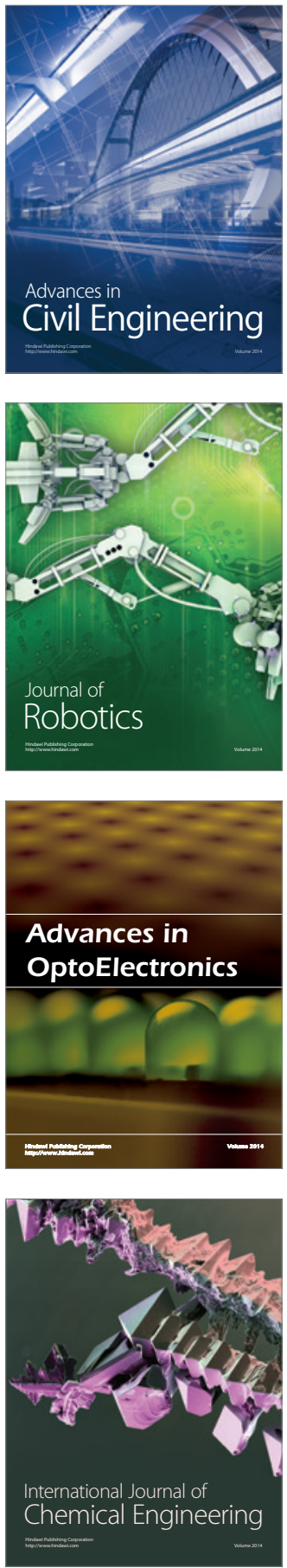

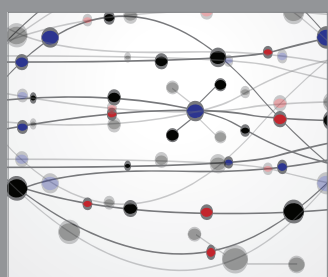

The Scientific World Journal

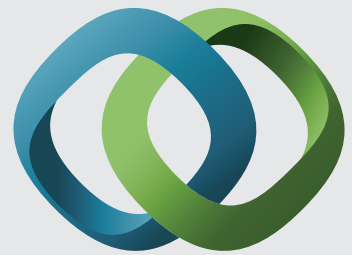

\section{Hindawi}

Submit your manuscripts at

http://www.hindawi.com
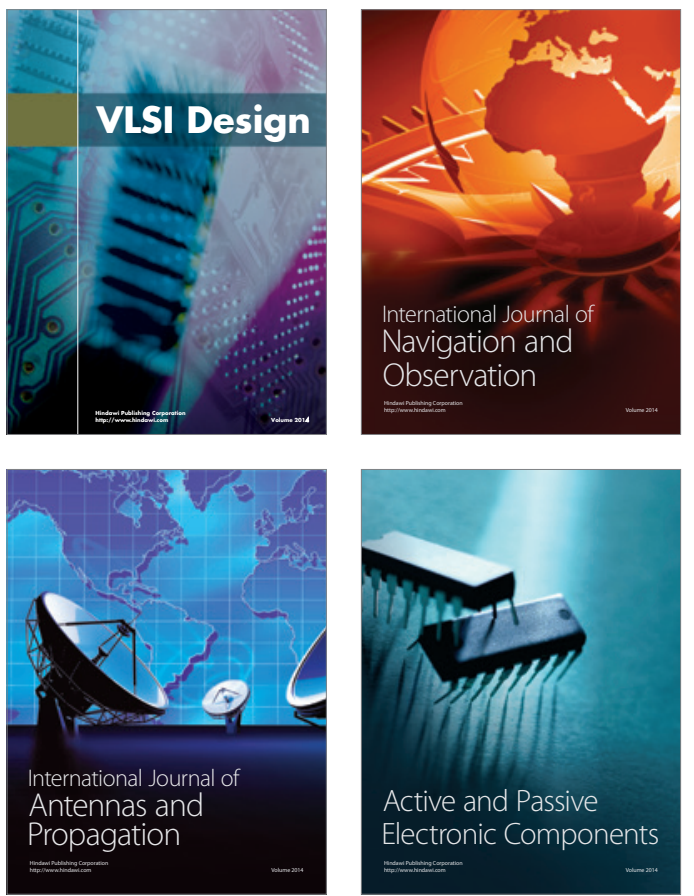
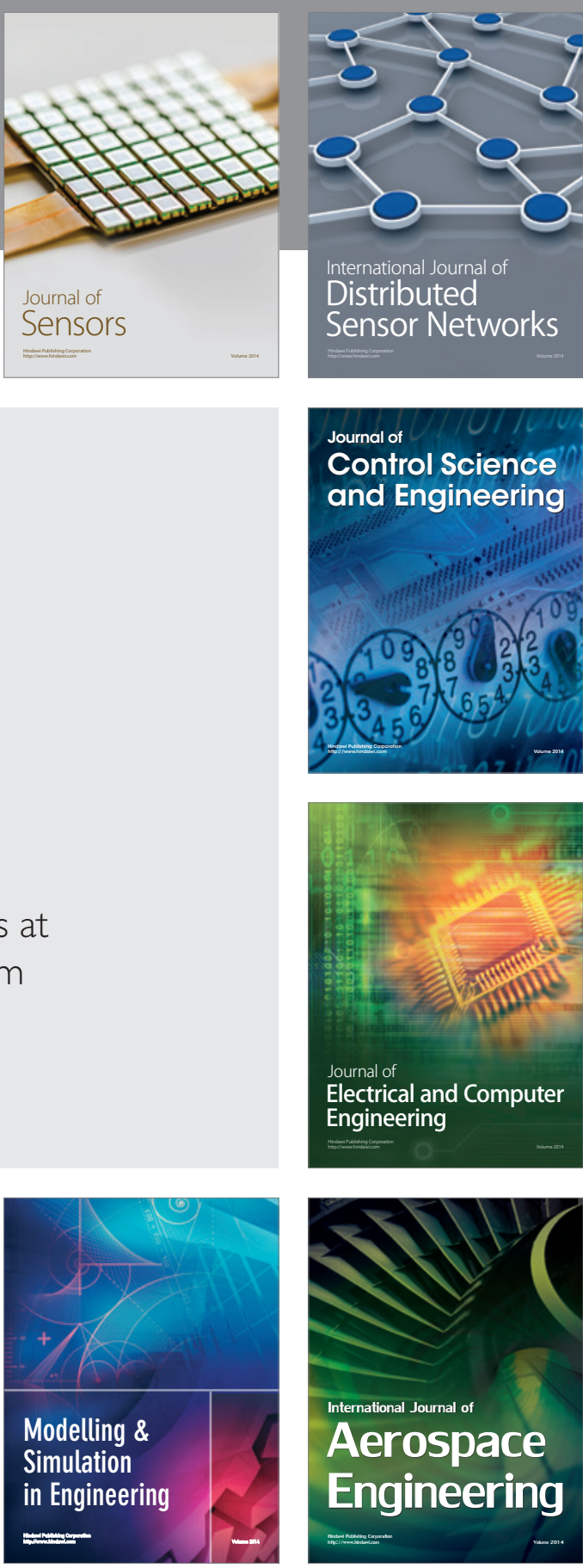

International Journal of

Distributed

Sensor Networks

Journal of

Control Science

and Engineering
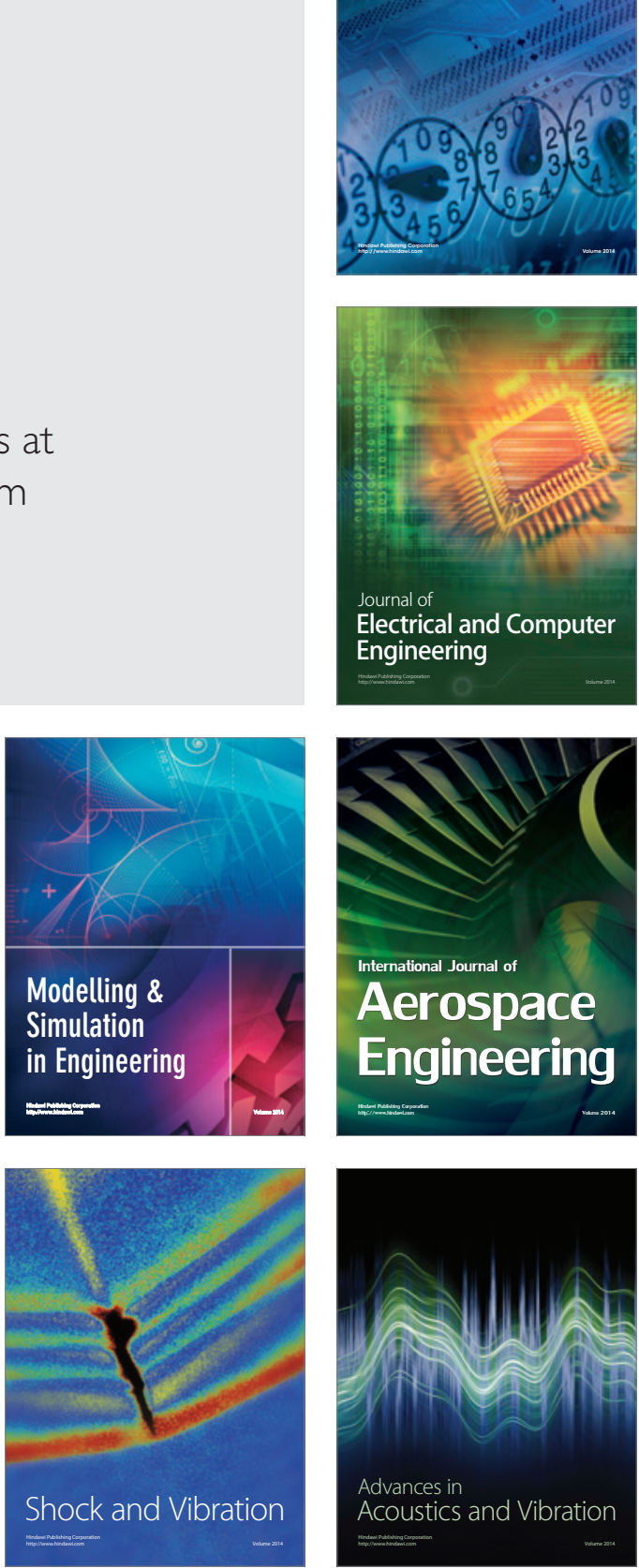